\title{
MR Brain Image Edge Detection Guided with Distribution of Noise and Artifact
}

\author{
Yuchou Chang \\ Computer Science and Engineering Technology Department, University of Houston-Downtown, Houston, USA
}

Email address:

changy@uhd.edu

\section{To cite this article:}

Yuchou Chang. MR Brain Image Edge Detection Guided with Distribution of Noise and Artifact. American Journal of Computer Science and Technology. Vol. 1, No. 1, 2017, pp. 1-7. doi: 10.11648/j.ajcst.20180101.11

Received: October 26, 2017; Accepted: November 13, 2017; Published: December 20, 2017

\begin{abstract}
Magnetic resonance imaging (MRI) has revolutionized radiology in past four decades. MR image edge detection can identify anatomy boundaries and extract features for image analysis applications like segmentation and recognition of anatomy structures. Traditional MR image edge detection methods directly identify discontinuities in MR image domain without considering distribution of noise and aliasing artifact produced from MR scanner and reconstruction. It is difficult to suppress effects of noise and aliasing artifact during the edge detection process. In this project, a novel MR brain image edge detection method is proposed, which is based on parallel MRI reconstruction method. Distribution of noise and aliasing artifact is characterized by geometry factor map that also guides edge detection process for avoiding detection of noise and aliasing artifact. A collaborative learning strategy is applied on voting edges for producing the final edge detection. Experimental results show that the proposed method not only keep anatomy structure boundaries without missing edge components, but also avoid detection of noise and artifact with wrong edges.
\end{abstract}

Keywords: Edge Detection, Magnetic Resonance Imaging, Geometry Factor, Canny Edge Detector, Aliasing Artifact

\section{Introduction}

Edge detection is a basic digital image processing technique [1]. It contains a variety of mathematical models to identify points or segments in images where image intensities change or discontinue sharply. Magnetic resonance (MR) images [2, 3] enable radiologists to observe anatomical or physiology structures inside the human body. A number of MRI pulse sequences [4] can be grouped to form a protocol with changing magnetic gradients and other parameters. Different types of anatomy or physiology tissues display different intensities on MR images, which are produced by a group of MR scan protocols. For this reason, MR image edge detection is not only dependent on traditional digital image processing techniques, but also related to imaging protocols and MR physics [5].

MRI noise [2, 3] may deteriorate edge detection performance, since many edge detection algorithms are sensitive to noise in the image. Noise can also degrade edge detection accuracy in MR images. Noise generation in MR scan is complicated, which is related to electrical current, coils, and magnetic field. Noise should be considered in MR image edge detection for suppressing noises and producing accurate edges. For traditional methods, filtering or smoothing is applied on detecting edges. It not only suppresses noises in the MR images, but also blurs anatomy tissue boundaries. For this reason, it may miss existing edges among different types of tissues.

Furthermore, aliasing artifact $[2,3]$ is caused when the field of view (FOV) is smaller than anatomy part during imaging. The artifact is wrapped around on the other side of the image. Aliasing artifact deteriorates edge detection performance, since its values change abruptly in neighbored pixels. It is easily detected by edge detection algorithms as the fake edges in image. Aliasing artifact is not true edges of different types of tissues, unlike edges around anatomy structure. Therefore, aliasing artifact should be eliminated, suppressed, or avoided in edge detection algorithms for MR images.

In this paper, an edge detection algorithm is proposed for a parallel MR imaging technique - Sensitivity Encoding (SENSE) [6], which has been implemented on almost all commercial MR scanners and widely used for clinical applications as shown in the Table 1. The method is 
different from traditional methods which denoise images to avoid edge detection accuracy caused by noise degradation. It also measures locations of aliasing artifact and eliminates wrong edge detection caused by aliasing artifact. The proposed method investigates noise generation in a parallel MR imaging technique and noise distribution characterized by geometry factor map. The introduction and background are presented at first. The proposed method will be illustrated in the third section. Results are evaluated and presented in the fourth part. Conclusion is given in the final section.

\section{Background}

Edge detection algorithms can be divided into gradient and Laplacian categories. The first category measures minimum and maximum in the first derivative of pixels in image, and the second category focuses on the second derivative of pixels in image. The Canny edge detector was proposed in 1986 [7], which extract structural information in visual objects. The typical Canny edge detection algorithm contains the following five steps [7]:

(1). Smoothing image for eliminating noise;

(2). Calculating intensity gradients of pixels;

(3). Applying non-maximum suppression to get rid of spurious response to edge detection;

(4). Determining potential edges by double thresholds;

(5). Tracking edge by hysteresis.

A number of methods have been proposed for MR image edge detection. SUSAN and Soble [17] edge detection were compared in MRI images for feature extraction [8]. It provided a guide to choose SUSAN or Soble edge detection in MRI feature extraction. In addition, optimal threshold is optimized for edge detection in MR brain images [9]. The adaptive threshold is determined by Ant Colony Optimization (ACO). The ACO based threshold outperforms other adaptive method. Different types of edge detection techniques were compared for brain MR images [10]. These edge detectors include Prewitt [17], Canny [7], Laplacian [18], and so forth. Results show that Canny edge detection technique extracts edges well from a brain MR image. On the other hand, edge detectors including Prewitt, Canny, Sobel, and Laplacian detect only local brightness transition with varying contrast or strength. From the global perspective of the entire image, when the threshold is too low, the detection result using these operators will produce more incorrect edges due to noise, or the correct edge cannot be detected when the threshold is too high. A novel algorithm for edge detection is proposed, in which global constraint and local contrast information are introduced to solve this problem [11]. It employed an ensemble of edge detectors that consider both local and global information. Results showed that the proposed method outperformed any single edge detector. This multi-view ensemble is also used in the proposed method of MR brain image edge detection.

Furthermore, MR image can be produced by fast parallel MR imaging technique to accelerate imaging speed. SENSE technique is one of widely used parallel MR image reconstruction technique. The fast speed of SENSE can make patients more comfortable and reduce clinical costs. Although, more noise and aliasing artifacts are produced as a tradeoff of imaging acceleration in SENSE method, it has been widely used in clinical applications. Table 1 shows acronyms [12] of SENSE implementation on five MR scanner vendors. For arbitrary trajectories, the general SENSE equation is

$$
\mathrm{Ef}=\mathrm{d}
$$

, where $\mathrm{d}$ is the vector formed from the $\mathrm{k}$-space data across all channels, $f$ is the full FOV image needed to be solved, and $\mathrm{E}$ is the sensitivity encoding matrix. Using the unfolding operation, signal is separated for each pixel in the reduce FOV to produce non-aliased full-FOV image. In addition, geometry factor, a priori signal-to-noise (SNR) estimates and an important criterion for designing coil arrays, is provided by

$$
g(\rho)=\sqrt{\left[\left(\mathrm{S}^{\mathrm{H}} \Psi^{-1} \mathrm{~S}\right)^{-1}\right]_{\rho, \rho}\left(\mathrm{S}^{\mathrm{H}} \Psi^{-1} \mathrm{~S}\right)_{\rho, \rho}}
$$

where $\rho$ is pixel, $\mathrm{S}$ is the sensitivity matrix, and $\Psi$ is the receiver noise covariance matrix. Geometry related noise enhancement increases rapidly when reduction factor increases during parallel MR imaging. The proposed method identifies distribution of noise and aliasing artifacts by using geometry factor for enhanced edge detection of MR brain images.

Table 1. Acronyms of SENSE implemented on major MR scanner vendors.

\begin{tabular}{ll}
\hline MR Scanner Vendors & Acronyms of SENSE Implementation \\
\hline Siemens & mSENSE \\
General Electric (GE) & ASSET \\
Philips & SENSE \\
Hitachi & RAPID \\
Toshiba & SPEEDER \\
\hline
\end{tabular}

\section{Proposed Method}

The flowchart of the proposed method is presented in Figure 1. The proposed edge detection method is based on SENSE reconstruction and geometry factor map. SENSE reconstructs undersampled k-space signals faster than traditional full sampled data acquisition from MR scanners. Due to production of noise and aliasing artifacts, conventional edge detection methods identify inappropriate boundaries as anatomy edges. A multi-view edge detection strategy is proposed, which uses ensemble learning to detect distribution of noise and aliasing artifacts from multiple windows. The details of multi-view mechanism are presented in the next section. 


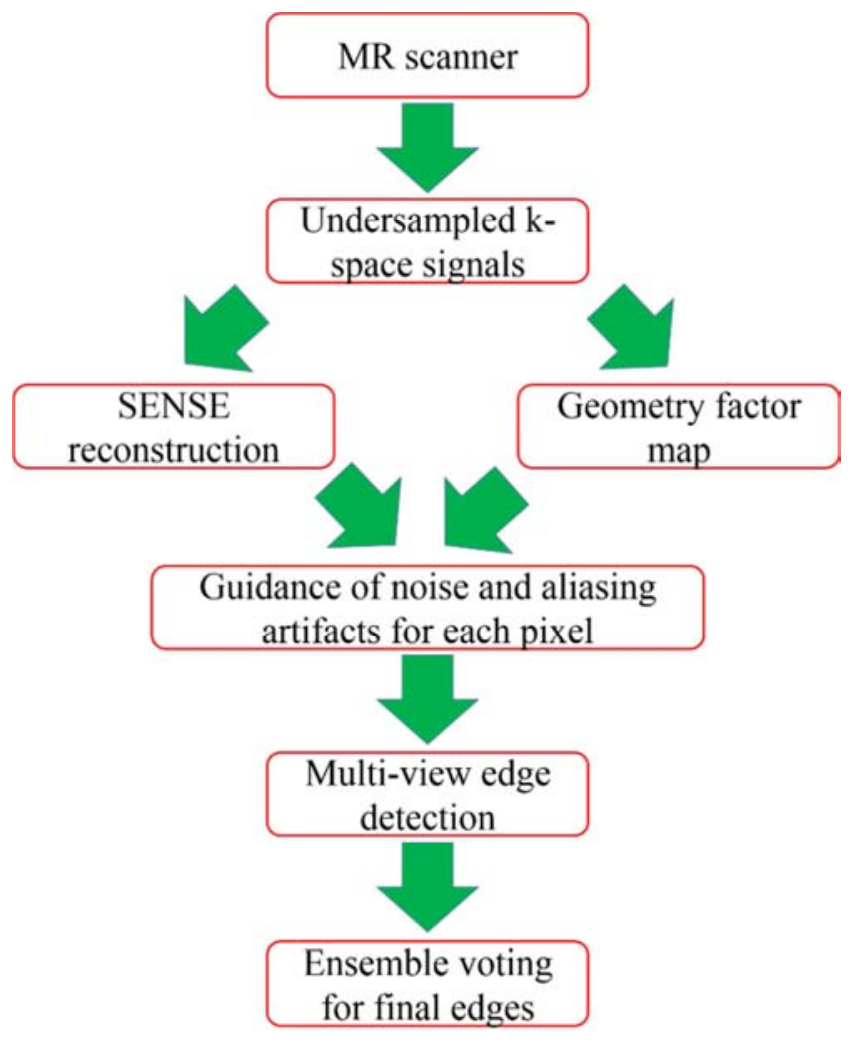

Figure 1. Flowchart of the proposed method.

\subsection{SENSE Reconstruction and Geometry Factor Map}

SENSE is a typical image-based parallel MRI reconstruction method. It makes a un-aliasing process in the image domain. After inverse Fourier transform of the $k$-space data, SENSE reconstructs aliased coil images. In the inverse Fourier transform process, the field of view (FOV) is reduced by the $1 / R$ ( $R$ is the reduction factor for undersampling $\mathrm{k}$ space). This means that the same information is contained in a smaller area which leads to fold over type aliasing artifacts. Following the equation (1), a brain MR image is reconstructed by SENSE reconstruction as shown in Figure 2 (d). It is seen that the reconstructed image deteriorates by noise and aliasing artifacts due to trade-off acceleration by undersampling signals on k-space. Furthermore, a geometry factor map is also produced as shown in Figure 1 (e). It can characterize distribution of noise and aliasing artifacts presented in SENSE image in Figure 1 (d). The higher values of geometry factor represents lower SNR in the corresponding pixel position in the image. The geometry factor is able to guide noise strength in the reconstructed SENSE image. The proposed method exploits geometry factor values for guiding canny edge detector in the image domain. Noise and aliasing artifacts should not be detected by edge detector.

\subsection{Edge Detection Via Multiple Views Ensemble}

The noise level of each pixel on reconstructed MR images can be characterized by geometry factor value. The higher value represents noise is stronger and SNR is lower. Each pixel is considered as the central point of the local window for edge detection calculation. However, windows size is generally fixed for different types of edge detectors. In the proposed method, Canny edge detector is executed in a window with random size and random pixel positions for detecting local edges. The proposed algorithm comes from collaborative learning of knowledge creation communities. The use of collaborative learning $[19,20]$ helps to determine the noise level of each pixel from multiple perspectives. This is similar to the previous work on image enhancement collaborative learning via multiple views [13].

Collaborative learning is firstly studied in education, which contains a group of approaches involving joint intellectual effort by students, teachers, and scholars [14]. Learners coordinate and adjust their methods and activities to enhance the knowledge construction and problem solving ability. The theory of collaborative learning is extended to machine learning research. For example, a refinement of classification hierarchies was proposed to use different kinds of clustering results for ensemble learning [15]. This method associates multiple instances of learning methods-clustering algorithms to achieve an optimally accurate classification. Inspired by the collaborative learning idea applied in machine learning research, we propose a MR brain image edge detection algorithm based on identifying distribution of noise and aliasing artifacts.

For a reconstructed MR image $I$ with the size of $W \times H$, the central point of the $i^{\text {th }}$ randomly chosen window is selected as following.

$$
\operatorname{Cen} X(i)=\operatorname{rand} \times W
$$

and

$$
\operatorname{Cen} Y(i)=\operatorname{rand} \times H
$$

where rand is a random real number generated between 0 and 1. The center of any window can be pixel position in the image. The width and height of the $i^{\text {th }}$ window are calculated as following.

$$
\operatorname{Win} W(i)=2 \times\left\lceil\frac{\operatorname{rand} \times W}{2}\right\rceil+1
$$

and

$$
\operatorname{Win} H(i)=2 \times\left\lceil\frac{\operatorname{rand} \times H}{2}\right\rceil+1
$$

$\lceil\cdot\rceil$ is the operation rounds the element to the nearest integer of the original value. Note that rand in Equations (3) to (6) produces a random number for each equation and for each window. Based on the center $(\operatorname{CenX}(i)$, CenY $(i))$ of the $i^{\text {th }}$ window, a window $S^{(i)}{ }_{W i n W(i) \times W i n H(i)}$ can be extracted from the original image $I$. For each window whose boundary exceeds the border of the image $I$, the portion of the window outside the original image is cut off. 
Since a pixel with higher geometry factor value represents higher level of noise, the window center pixel's geometry factor value serves as an indicator of the local window noise levels. For a local window whose center pixel has higher geometry factor value, a higher threshold is used for Canny edge detection to suppress noise. On the other hand, for a window whose center pixel has lower geometry factor value, a lower threshold values is applied on Canny edge detector for detecting local edges within each window.

\subsection{Edge Detection Ensemble Via Local Detectors' Voting}

Classifier ensembles and clustering ensembles have been widely studied in machine learning research. Robust and accurate results can be obtained by ensemble of individual classifiers or clusters. Voting has been proven to be effective in many classification or clustering applications. For example, cumulative voting was proposed to solve the problem of cluster label alignment $[16,21]$. Accuracy can be improved in compared to using individual clustering algorithms. The voting is also applied in the proposed method for detecting true edges generated from multiple windows.

\section{Results}

The proposed algorithm is evaluated on two MR brain image datasets. One is axial brain image dataset and another one is sagittal brain image dataset. The axial image dataset contains 8 channels acquired from 8 receiver coils and the sagittal dataset includes 4 channels acquired from 4 receiver coils. The sensitivity map is calculated based on the central k-space data, which is fully sampled. The reduction factor is 4 for undersamplilng $\mathrm{k}$ space data for both brain image datasets. The number of windows is set up as 200 and threshold of Canny edge operator is fixed as 0.05 for both of reference image and SENSE image. For the proposed method, the threshold of Canny edge operator ranges from 0.05 to 0.3 . Each window's threshold is determined by geometry factor value on the center pixel of that window.

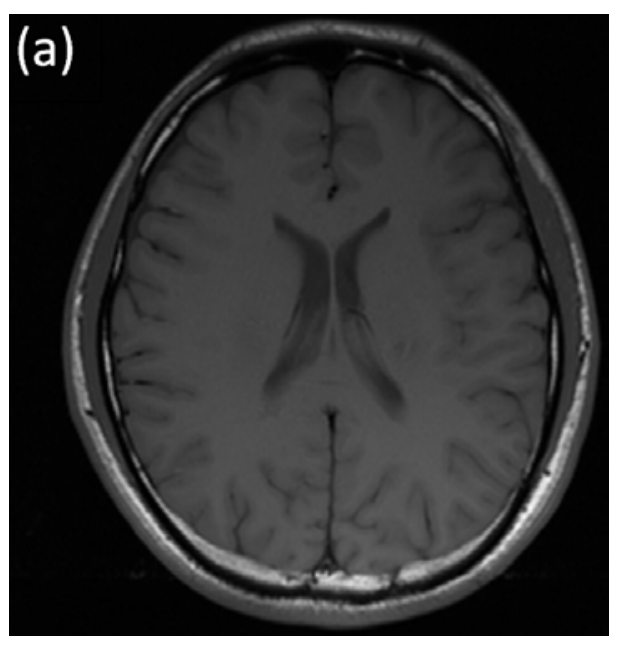

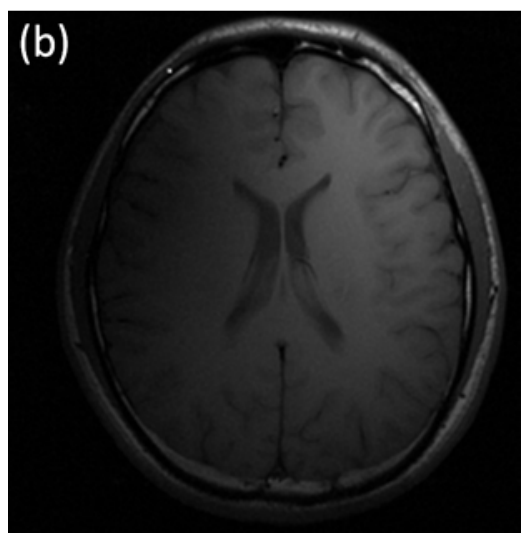
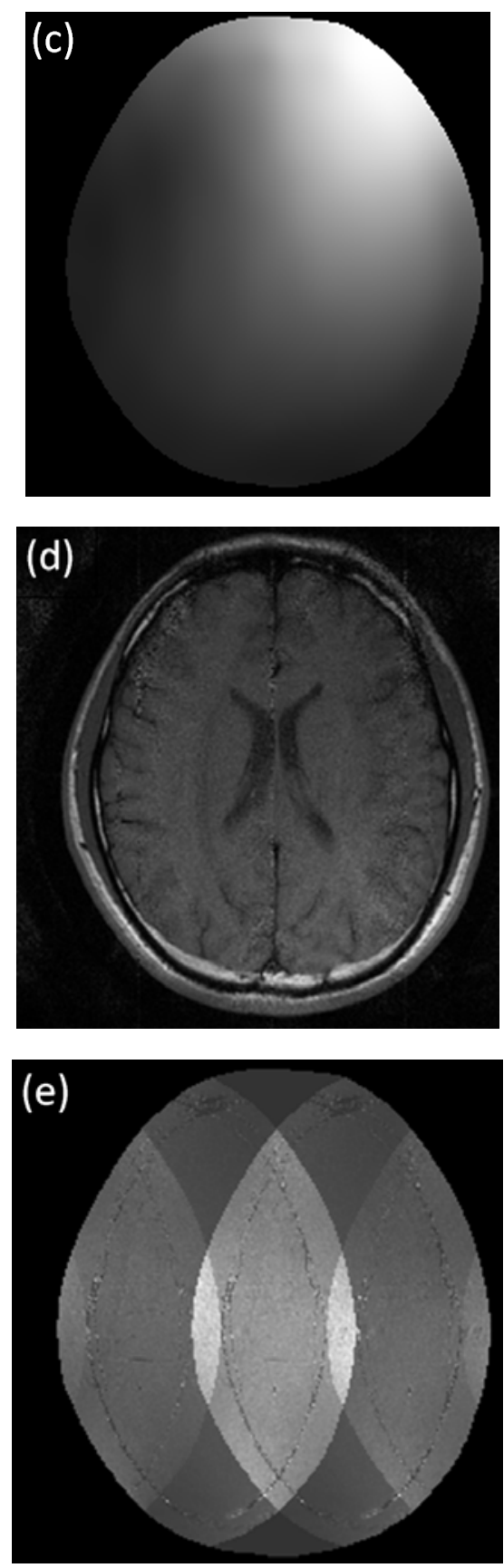

Figure 2. Reference image (a) of the first acquired data with 8 coils, one coil image (b) and its coil sensitivity map (c), as well as SENSE reconstruction (d) and its geometry factor map (e). 
The reference is calculated from traditional canny edge detection on fully sampled reconstruction. As shown in the Figure 2 (a), the reference image has few noise and aliasing artifacts. It is fully sampled but required longer acquisition time on MR scanner. One coil image and its coil sensitivity map is presented in Figure 2 (b) and (c). The coil sensitivity produces noise and aliasing artifacts in the reconstructed SENSE image, thereby reducing image quality. It can be seen that edge detection on the reference image can detect most of the boundaries between white matter, gray matter and cerebrospinal fluid (CSF). Traditional canny detector is also applied on brain MR image with noise and aliasing artifacts, which is reconstructed by SENSE. The corresponding geometry factor map indicates distribution of noise and aliasing artifacts. The traditional canny detector on SENSE image presents contradiction of missing edges and wrong edges. As shown in Figure 3 (c), brain boundaries indicated by green arrow are missed by canny detector, but aliasing artifact indicated by red arrow is wrongly detected. For the proposed algorithm, aliasing artifact is suppressed without detection in Figure 3 (d). It is closer to Figure 3 (b) of edge detection on reference image without noise and aliasing artifact. Furthermore, brain boundaries are detected correctly, which are similar to edges detected in the reference image.
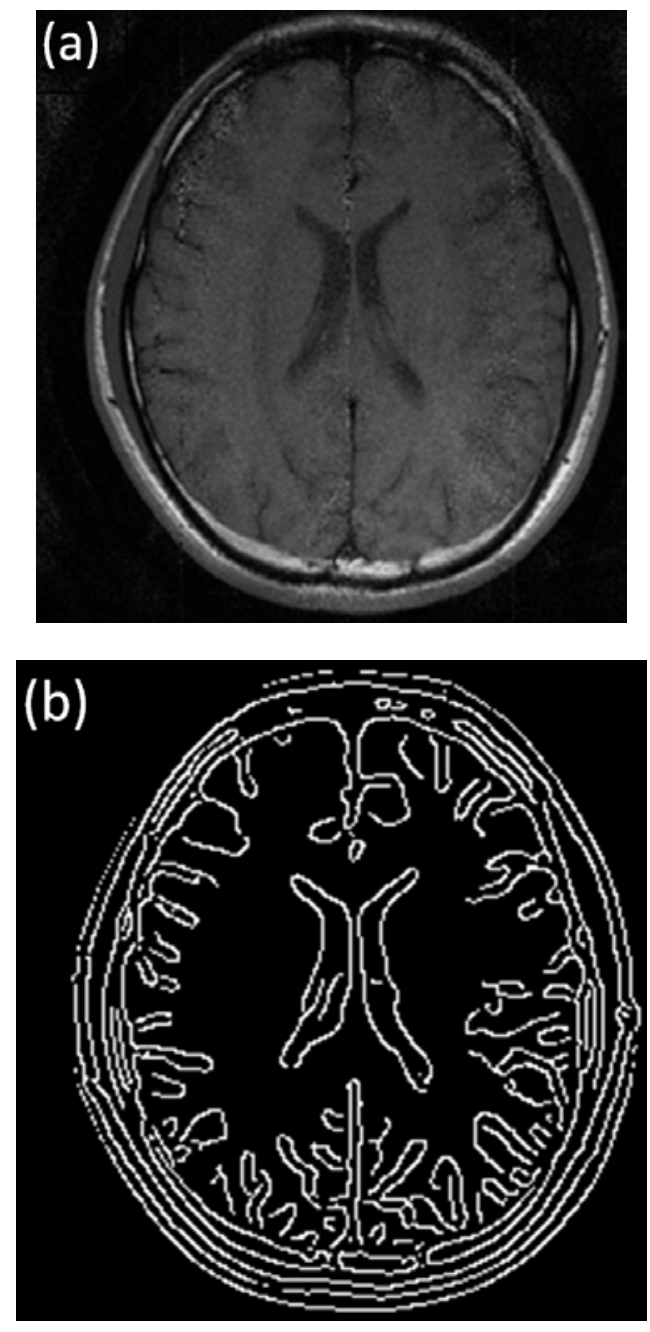
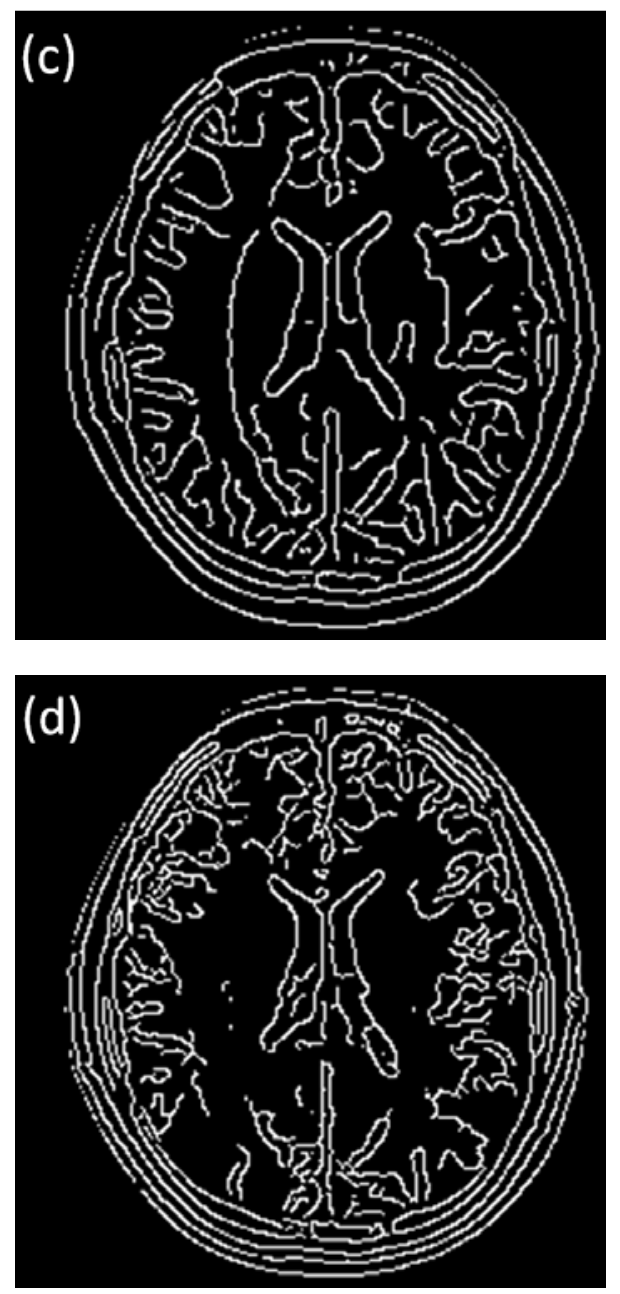

Figure 3. Reference MR brain image (a) of the first dataset, Canny edge detection (b) on reference image (a), Canny edge detection (c) on SENSE image (Figure $2(d)$ ), and proposed method (d) on SENSE image (Figure 2 (d)).

The proposed method is applied on another MR brain image as shown in Figure 4. The reference is also calculated from the traditional canny edge detection of the complete sampling reconstruction in Figure 4 (a). As shown in Figure 4 (a), the reference image has little noise and aliasing artifacts since it is fully sampled. A coil image and its coil sensitivity diagram shown in Figure 4 (b) and (c). The SENSE image and its geometry factor map are shown in Figure 4 (d) and (e). It can be seen that edge detection on the reference image can detect most of the margins between anatomy structures. Conventional canny detectors are also applied to brain MR images with noise and aliasing artifacts reconstructed by SENSE. The corresponding geometric factor assists edge detection of the proposed method. SENSE images on the traditional canny detectors exist missing edges and edges of the error. As shown in Figure 5, the proposed method (Figure 5 (d)) avoids noise and aliasing artifacts such that the edges cause the brain boundary to be correctly detected, which is similar to the edge detected in the reference image (Figure 5 (b)). On the other hand, as shown in Figure 5 (c), the conventional canny operator cannot suppress noise and aliasing artifacts, thus producing more extraneous edges. 

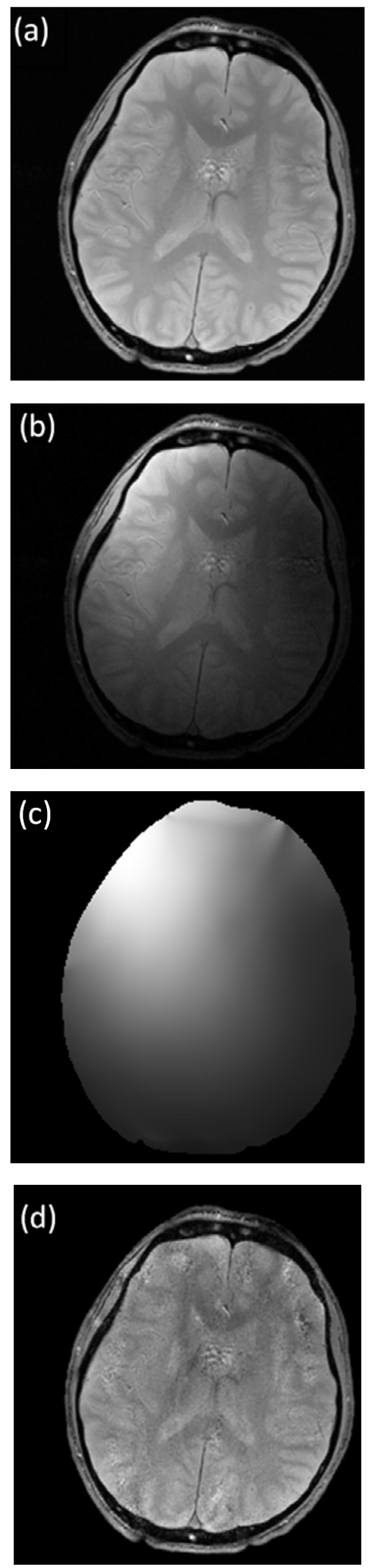

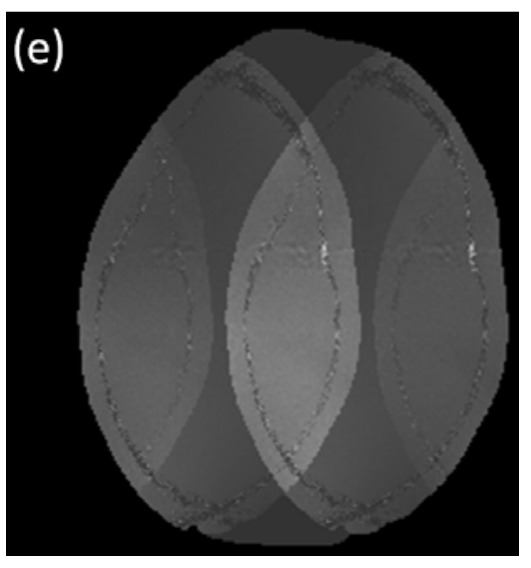

Figure 4. Reference image (a) of the second acquired data with 8 coils, one coil image (b) and its coil sensitivity map (c), as well as SENSE reconstruction (d) and its geomentry factor map (e).
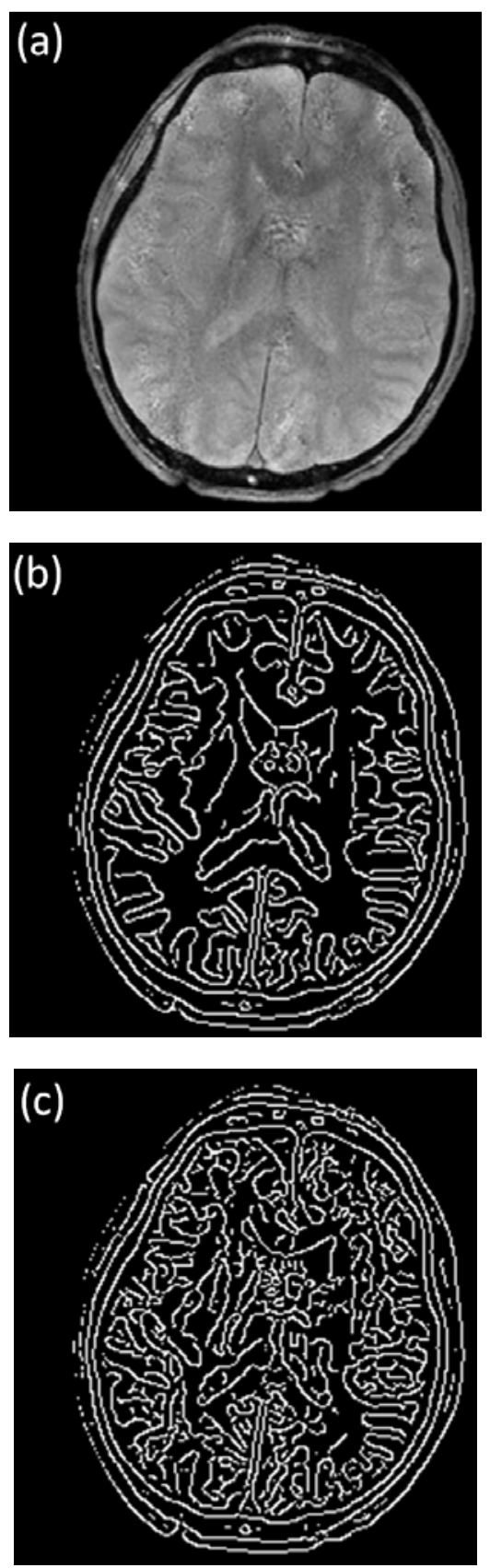


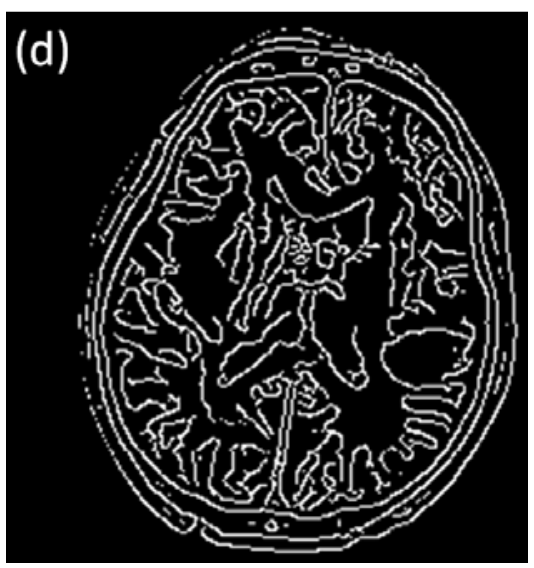

Figure 5. Reference MR brain image (a) of the second dataset, Canny edge detection (b) on reference image (a), Canny edge detection (c) on SENSE image (Figure $4(d)$ ), and proposed method (d) on SENSE image (Figure 4 (d)).

\section{Conclusion}

A new MR image edge detection method has been proposed based on distribution of noise and aliasing artifacts. First, noise and aliasing artifacts are characterized by producing geometry factor map with SENSE parallel MRI reconstruction. Then, a strategy of using multiple windows with randomly chosen sizes and centers is used to simulate individuals of the learning community for detecting edges in each window. Edge detection on each local window is guided by geometry factor value. Final, global edges is produced by counting votes from edge detections in multiple windows. The experimental results show that this method not only improves the accuracy of the global edge of the whole image, but also eliminates the incorrect edges caused by noise and aliasing artifacts.

\section{References}

[1] Jain, A. K.: Fundamentals of Digital Image Processing. Prentice-Hall Information And System Sciences Series (1989).

[2] Z. P. Liang, and P. C. Lauterbur, "Principles of magnetic resonance imaging: a signal processing perspective", WileyIEEE press, 1999.

[3] D. B. Twieg, "The k-trajectory formulation of the NMR imaging process with applications in analysis and synthesis of imaging methods", Medical Physics, vol. 10, pp. 610-621, 1983.

[4] Kevin F. King, Matt A. Bernstein, and Xiaohong Joe Zhou. "Handbook of MRI pulse sequences". Academic Press, 1st edition, 2004.

[5] E. Mark Haacke, Michael R. Thompson, Ramesh Venkatesan, Robert W. Brown, and Y.-C. Norman Cheng. "Magnetic resonance imaging: Physical principles and sequence design". Wiley-Liss, 1st edition, 1999.

[6] Klaas P. Pruessmann, Markus Weiger, Markus B. Scheidegger, and Peter Boesiger, "SENSE: Sensitivity Encoding for Fast MRI", Magnetic Resonance in Medicine, vol. 42, pp. 952-962, 1999.
[7] Canny, J.: A Computational Approach to Edge Detection. IEEE Transactions on Pattern Analysis and Machine Intelligence 8 (6), 679-698 (1986).

[8] G. Rezai-Rad; M. Aghababaie. Comparison of SUSAN and Sobel Edge Detection in MRI Images for Feature Extraction. Information and Communication Technologies, 2006.

[9] Jansi, S.; Subashini, P. Optimized Adaptive Thresholding based Edge Detection Method for MRI Brain Images. International Journal of Computer Applications, vol. 51, issue 20, pp. 1-8, 2012.

[10] A. V. Kavitha, Madhulika. Y, Siddardha Kumar. M. Comparison of Edge Detection Techniques for Brain MRI Image. International Journal of Com puter Science and Information Technology \& Security (IJCSITS), ISSN: 22499555 Vol. 5, No 3, June 2015.

[11] Yuchou Chang, Dah-Jye Lee, Yi Hong, and James K. Archibald, "Edge Detection from Global and Local Views Using an Ensemble of Multiple Edge Detectors", International Symposium on Visual Computing, pp. 934-941, Las Vegas, Nevada, United States, Dec. 1-3, 2008.

[12] MRI Acronyms. URL: https://ibiswiki.cit.nih.gov/foswiki/pub/Main/DataElements/ImagingData Elements/MRI_AcronymsSiemenceandGE.pdf.

[13] Yuchou Chang, Dah-Jye Lee, James K. Archibald, and Yi Hong, "Using Collaborative Learning for Image Contrast Enhancement", IEEE Conference on Pattern Recognition (ICPR), Tampa, Florida, United States, Dec. 8-11, 2008.

[14] A. M. O'Donnell, C. E. Hmelo-Silver, and G. Erkens, Collaborative Learning, Reasoning, and Technology, Lawrence Erlbaum Associates, Inc., New Jersey, ISBN: 0805-84778-2, September, 2005.

[15] C. Wemmert, P. Gancarski, and J. Korczak, An Unsupervised Collaborative Learning Method to Refine Classification Hierarchies, IEEE International Conference on Tools with Artificial Intelligence, pp. 263- 270, 1999.

[16] Avad, H. G., and Kamel, M. S., Cumulative Voting Consensus Method for Partitions with Variable Number of Clusters, IEEE Transactions on Pattern Analysis and Machine Intelligence, vol. 30, no. 1, pp. 160-173, 2008.

[17] Gonzalez, R. C., and Woods, R. E., Digital Image Processing, Publisher: Addison Wesley, ISBN: 0-201-50803-6, 1992.

[18] Berzins, V., Accuracy of Laplacian Edge Detectors, Computer Vision, Graphic and Image Processing, vol. 27, no. 2, pp. 195$210,1984$.

[19] Xu, K., Ding, H., Guo L., Fang, Y., “A Secure Collaborative Machine Learning Framework Based on Data Locality", IEEE Global Communications Conference (GLOBECOM), 2015.

[20] Rao, M., "A Collaborative Filtering Recommender System with Randomized Learning Rate and Regularized Parameter", IEEE International Conference on Current Trends in Advanced Computing (ICCTAC), 2016.

[21] Wei, X., Cao, B., and Yu, B., "Multi-View Unsupervised Feature Selection by Cross-Diffused Matrix Alignment", International Joint Conference on Neural Networks (IJCNN), 2017. 